\title{
Perioperative Challenges in Patients with Alpha-Gal Allergy
}

\author{
Francine J D'Ercole, $M D^{1 *}$, Vishal H Dhandha, MD, MHA ${ }^{1}$, Marc L Levi, $M D^{1}$, April B Todd, $R N^{2}$ and Priya \\ A Kumar, $M D^{1}$
}

${ }^{1}$ Department of Anesthesiology, University of North Carolina, Chapel Hill, NC, USA

${ }^{2}$ Perioperative Nursing, University of North Carolina, Chapel Hill, NC, USA

\begin{abstract}
Alpha-gal allergy, also known as, mammalian meat allergy (MMA) is well described in the Allergy literature, however, the Anesthesiology literature remains soft in supporting recommendations for perioperative management. The goal of the Centers for Disease Control and Prevention (CDC) is to better understand the pathogenesis, signs and symptoms, and prevention of this delayed anaphylactic reaction compared to the rapid onset of most food allergies, especially immunoglobulin E (IgE) mediated allergy. MMA is not limited to dietary beef, bison, goat, pork, lamb, and venison intake alone but includes some perioperative medication formularies containing inactive ingredients such as gelatin, glycerin or stearate; surgical products such as surgical powder, xenografts, and porcine derived heart valve per the manufacturer's specifications. This report will include the brief of 6 patients with alpha-gal allergy who presented during 2018 for elective surgery at a North Carolina community hospital when no patients prior were identified with MMA. The patients described have demonstrated one or more of the several perioperative challenges unique to alpha-gal allergy outlined in this manuscript. Authors have identified eight challenges representing knowledge gaps impacting safe anesthesia care.
\end{abstract}

\section{Introduction}

Delayed anaphylaxis to mammalian meat in patients with IgE antibodies specific for galactose-alpha-1,3-galactose (alpha-gal) has been described by Commins and Platts-Mills $[1,2]$. The carbohydrate alpha-gal is found in the meat of non-primate mammals, cow's milk, and cat and dog dander. Additionally, delayed allergic reaction to alpha-gal includes some perioperative medication formularies containing inactive ingredients such as gelatin, glycerin or stearate, as well as, bovine or porcine prepared heparin, biologic mesh, catgut suture, surgical powder, xenografts, and porcine derived heart valve. Increasing awareness and knowledge regarding alpha-gal allergy is the first step in the selection of anesthetic agents or providing anesthesia techniques that will not trigger an immune response.

To better understand this relatively new alpha-gal allergy and to provide safe anesthesia care during the perioperative period an initial literature search was performed on PubMed. Relevant articles providing an important historical perspective and identifying knowledge gaps existing in the perioperative process were selected. A systematic review was performed, and relevant references from the initial search articles were selected. Additionally, the authors accessed the CDC website and added important links, including the means to access for future updates.

\section{Alpha-Gal Allergy \\ Discovery}

Interestingly, Platt-Mills himself was bitten by a tick and developed the alpha-gal allergy providing the link (in 2002) between the allergy and the tick vector [3]. There are two types of anaphylaxis triggered by alpha-gal: Delayed-type and immediate type. In 2004, the monoclonal chimeric antibody cetuximab (Erbitux) was in clinical trials for metastatic colorectal cancers resulting in a considerable number of patients (15\%) immediately reacting to the first dose with severe and often fatal anaphylaxis.

Commins and Platts-Mills were trying to understand why some people were reacting to the carbohydrate cetuximab. In 2007, the rates of hypersensitivity reactions in patients treated with cetuximab in the Northeast US were reported to be $<1 \%[4,5]$. However, when compared to Tennessee and North Carolina severe hypersensitivity reactions were reported to be $22 \%$ in patients treated with cetuximab [4]. Chung, et al. in 2008 reported that, in healthy control subjects from the Tennessee group, $20.8 \%$ had preexisting

*Corresponding author: Francine J D'Ercole, Department of Anesthesiology, University of North Carolina, N2198 UNC Hospitals, Campus Box 7010, Chapel Hill, NC 27599, USA

Accepted: June 20, 2019

Published online: June 22, 2019

Citation: D’Ercole FJ, Dhandha VH, Levi ML, et al. (2019) Perioperative Challenges in Patients with Alpha-Gal Allergy. J Clin Anesth Pain Manag 3(1):70-78

Copyright: (c) 2019 D'Ercole FJ, et al. This is an open-access article distributed under the terms of the Creative Commons Attribution License, which permits unrestricted use, distribution, and reproduction in any medium, provided the original author and source are credited. 
IgE antibodies against an oligosaccharide present on the recombinant cetuximab molecule [5]. Striking differences in the prevalence of this antibody were found in the Northern California (6.1\%) and the Boston (0.6\%) groups.

In 2011, a second type of (delayed) anaphylaxis related to the presence of IgE antibodies to the oligosaccharide alphagal was described in an investigation by Commins, et al. [6]. Results in Virginia, North Carolina, and Tennessee revealed that as many as $20 \%$ of the population in these states have serum IgE antibodies to alpha-gal. This strongly suggested tick bites as a cause of IgE antibody responses to alpha-gal in the southeastern United States [6].

Humans who test positive for alpha-gal IgE have been sensitized to alpha-gal after a tick bite, usually species specific in the United States and Australia (Table 1) [7]. In addition to alpha-gal allergies representing the first food allergies presenting with delayed anaphylaxis, MMA is the first known food allergy triggered by a carbohydrate alpha-gal oligosaccharide antigen. In addition to mammalian meat and cetuximab, there is increasing concern for allergic reactions to other commonly used perioperative medications, surgical products, and surgical devices containing this alpha-gal trigger.

\section{Mechanism}

Alpha-gal is not inherently present in humans or primates compared to all other mammals. If a species-specific tick feeds on a mammal, the alpha-gal carbohydrate remains in the tick's alimentary tract and saliva [8-11]. The tick vector is capable of injecting alpha-gal into a human, triggering an immune response of IgE antibodies against the foreign carbohydrate. Future intake of mammalian meat (beef, pork, lamb) results in an amplified response, producing symptoms caused by an over-abundance of IgE antibodies attacking the alpha-gal antigen. Since serological conversion to alpha-gal takes several months symptoms may be delayed for three to six months after the tick bite.

\section{Diagnosis}

Traditional thinking is that humans become sensitized to foods during childhood and subsequent exposure to certain

Table 1: Profile of Alpha-gal Allergy.

\begin{tabular}{|l|l|}
\hline Vectors [7-10] & $\begin{array}{l}\text { Amblyomma americanum also known as (aka) Lone star tick, Northeastern water tick, turkey tick in the eastern United } \\
\text { States, and Mexico }\end{array}$ \\
\hline & $\begin{array}{l}\text { Ixodes holocyclus aka Paralysis tick Australian species } \\
\text { Ixodes ricinus aka Castor bean tick European species }\end{array}$ \\
\hline Other existing unknown vectors \\
\hline Symptoms & Anaphylaxis, angioedema, urticaria, gastrointestinal disturbance \\
\hline Young age, suggestive of other unknown ways of sensitization \\
\hline Diagnosis & Assay measuring IgE specific to alpha-gal (Viracor-IBT Labs) \\
\hline Skin test, Basophil activation test with cetuximab (more reliable) > meats \\
\hline Treatment & Delayed life-threatening symptoms: epinephrine, steroids, H-2 blockers \\
\hline Cure & None \\
\hline Duration & Unconfirmed recovery potential \\
\hline
\end{tabular}

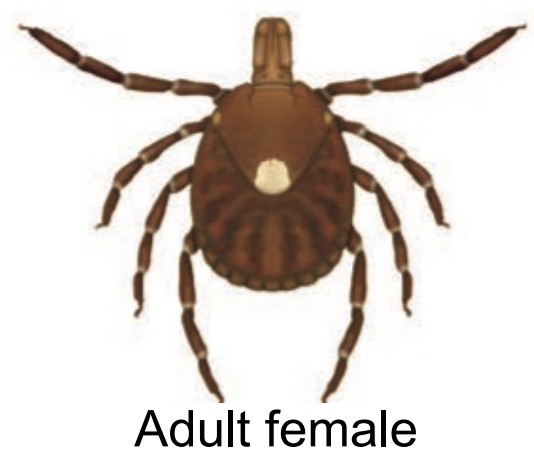

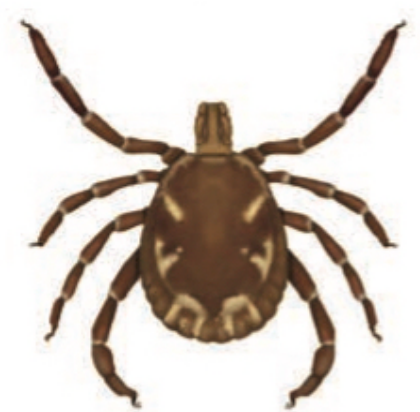

Adult male
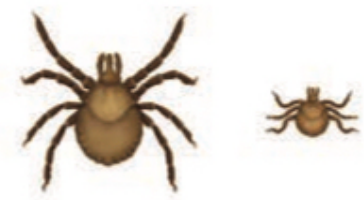

Nymph Larva

Figure 1: Adapted from image available from Center for Disease Control and Prevention in the public domain [7].

Comments: The adult female is distinguished by a white dot or "lone star" on her back. The nymph and adult females most frequently bite humans and transmit disease. The species is considered very aggressive.

United States distribution: Eastern and southeastern United States.

Causation and Transmission: Alpha-gal, Human Ehrlichiosis, Tularemia, and Southern Tick Associated Rash Illness (STARI). 
foods triggering an immediate allergic response. Alpha-gal allergy represents the first identified delayed onset IgE anaphylaxis making it more difficult in determining the link between the food allergen and the allergic reaction.

Kennedy JL, et al. determined from clinics or offices in the state of Virginia IgE antibodies to alpha-gal were present in the serum of pediatric patients having a history of delayed anaphylaxis or urticaria to mammalian meat with most cases having a history of tick bites within the past (one) year [12]. Since 2013, other reports have been consistent that the production of IgE antibodies to alpha gal is triggered by a bite from Amblyomma americanum or the lone star tick endemic to southeastern United States (Figure 1) [7,13-17].
Alpha-gal IgE assay is commercially available to detect specific IgEantibodies against alpha-1,3-galactose both highly sensitive and specific to IgE; $[18,19]$ however, usually purchased by private individuals. Blood testing for IgE response has not been approved by the United States Food and Drug Administration. (For information on Viracor-IBT Laboratories' testing visit: http://www.ViracorlBT.com).

The prevalence of sensitization to alpha-gal is unknown, but much more common than previously understood [6]. Skin-prick allergy test for MMA may give false-negatives [20]. Skin and basophil activation tests with cetuximabare the most sensitive, [21,22] but not cost-effective making availability limited (Table 1).

Table 2a: Case List.

\begin{tabular}{|c|c|c|c|}
\hline Case \# & Demographics & Anesthetic evaluation or pertinent history & Surgical and perioperative treatments \\
\hline Case 1 & $\begin{array}{l}\text { 74-year-old male, retired } \\
\text { professional healthcare } \\
\text { worker. }\end{array}$ & $\begin{array}{l}\text { Patient reported }>30 \text { anaphylactic } \\
\text { reactions between } 2015-2018 \text {. Reactions } \\
\text { included medications with hidden } \\
\text { ingredients containing mammalian } \\
\text { byproducts; cross-contaminated foods. }\end{array}$ & $\begin{array}{l}\text { General anesthesia for elective major cervical } \\
\text { spine surgery: Anterior cervical 4-7 decompression, } \\
\text { corpectomy, instrumented fusion with cages and } \\
\text { allograft; plate and screws. } \\
\text { Outsourcing food and meals from home. }\end{array}$ \\
\hline Case 2 & 59-year-old female & $\begin{array}{l}\text { Reported immediate anaphylaxis to the } \\
\text { cancer drug cetuximab. Delayed allergy } \\
\text { to beef and porcine containing products; } \\
\text { medications containing maltose. } \\
\text { Hydroxychloroquine induced Steven- } \\
\text { Johnson syndrome. }\end{array}$ & $\begin{array}{l}\text { General anesthesia for elective gynecologic surgery: } \\
\text { Hysteroscopy, polypectomy. }\end{array}$ \\
\hline Case 3 & 84-year-old female & $\begin{array}{l}\text { Reported positive formal allergy testing } \\
\text { for alpha-gal allergy. Reactions included } \\
\text { pruritis and urticaria after consuming beef } \\
\text { on several occasions. }\end{array}$ & $\begin{array}{l}\text { Neuraxial anesthesia for elective total knee } \\
\text { arthroplasty and received preoperative triggering } \\
\text { agents inadvertently via ERAS protocol. }\end{array}$ \\
\hline Case 4 & 37-year-old female & $\begin{array}{l}\text { Pertinent medical history included } \\
\text { comorbidities: Cushing's disease, diabetes } \\
\text { insipidus. Alpha-gal allergy was known by } \\
\text { patient but not updated in EHR. Patient } \\
\text { inadvertently received celecoxib and } \\
\text { gabapentin as part of an ERAS protocol } \\
\text { before her alpha gal allergy was realized. }\end{array}$ & $\begin{array}{l}\text { General anesthesia for elective total laparoscopic } \\
\text { hysterectomy and cystoscopy. The pharmacy } \\
\text { packaging for the drug did not have an NDC number. } \\
\text { The drug lot number proved to be insufficient for } \\
\text { providers to determine the manufacturer in a timely } \\
\text { fashion. Subsequently, preemptive steroids and } \\
\text { antihistamines were administered perioperatively. }\end{array}$ \\
\hline Case 5 & 60-year-old female & $\begin{array}{l}\text { Patient at high risk for recurrent DVT. } \\
\text { Reviewed the allergist's consult in } 2018 \\
\text { documenting } 50 \% \text { decrease in both alpha- } \\
\text { gal and IgE levels compared to previous } \\
\text { titers from } 2017 .\end{array}$ & $\begin{array}{l}\text { General anesthesia for emergent laparoscopic } \\
\text { cholecystectomy with known alpha-gal allergy. } \\
\text { After considering benefits versus risks the patient } \\
\text { received a potential triggering agent enoxaparin for } \\
\text { DVT prophylaxis prior to surgery and continued post- } \\
\text { operatively during hospitalization. }\end{array}$ \\
\hline Case 6 & 80-year-old female & $\begin{array}{l}\text { The patient reported an allergy to beef } \\
\text { and pork in the PACU. The allergy was } \\
\text { unclassified as alpha-gal allergy prior to } \\
\text { surgery. } \\
\text { Other allergies including anaphylaxis, hives } \\
\text { or swelling included: dextromethorphan, } \\
\text { hydrocodone, oxycodone, amoxicillin, } \\
\text { levaquin. }\end{array}$ & $\begin{array}{l}\text { General anesthesia for elective colpopexy, } \\
\text { extraperitoneal sling for stress incontinence, repair of } \\
\text { rectocele, cystoscopy. Patient did not qualify for an } \\
\text { ERAS protocol, therefore common triggering agents } \\
\text { were not introduced. Intraoperative management } \\
\text { included dexamethasone. In the PACU, the patient } \\
\text { developed anaphylaxis including bronchospasm, } \\
\text { diffuse rash over the chest and neck, and perioral } \\
\text { swelling immediately after receiving IV albumin for } \\
\text { fluid management and correction of hypotension } \\
\text { with intraoperative EBL } 125 \text { milliliters. Symptoms } \\
\text { improved after diphenhydramine IV. }\end{array}$ \\
\hline
\end{tabular}

ERAS: Enhanced Recovery After Surgery; HER: Electronic Healthcare Records; NDC: National Drug Code; DVT: Deep Venous Thrombosis; PACU: Postoperative Anesthesia Care Unit; IV: Intravenous. 


\section{Prognosis}

Recovery potential from this MMA-food allergy is not confirmed. Since eggs, poultry, and fish do not trigger the allergic reaction, individuals with the alpha-gal diagnosis do not need to become vegan or vegetarian.

\section{Description of 6 Case-Reports}

All cases presented were managed in a North Carolina community hospital. All patients confirmed a previous history of tick bites. Authors briefly describe 6 cases with different clinical presentations and perioperative concerns including differing sensitization characteristics. Every case either had a known increased risk for MMA or had a perioperative reaction with the discovery of an association to MMA, therefore, each case merits individual analysis (Table 2a Case list). Additionally a group analysis was conducted for patients who were at increased risk but did not experience manifestations of an alpha-gal allergic reaction despite receiving triggering agents either accidentally or intentionally (Table $2 b$ ).

\section{Case 1}

Describes a patient with a history of severe symptoms and recurrent anaphylaxis who employed a private pharma-

Table 2b: Cases without perioperative complications including patients at risk that either inadvertently or intentionally received triggering agents, example cases 3-5.

\begin{tabular}{|c|c|c|c|}
\hline $\begin{array}{l}\text { Case } \\
\text { number }\end{array}$ & Anesthetic agents or modalities & $\begin{array}{l}\text { Preemptive agents, ERAS and } \\
\text { Perioperative Analgesics }\end{array}$ & $\begin{array}{l}\text { Other drugs/surgical products } \\
\text { received }\end{array}$ \\
\hline 1 & $\begin{array}{l}\text { Endotracheal tube and IV agents: } \\
\text { midazolam, fentanyl, lidocaine, propofol } \\
\text { bolus, succinylcholine, rocuronium, } \\
\text { dexamethasone. } \\
\text { Neurophysiologic monitoring with TIVA: } \\
\text { propofol and remifentanvl infusions. } \\
\text { Invasive monitoring: Radial arterial line. }\end{array}$ & $\begin{array}{l}\text { Preemptive IV agents: } \\
\text { diphenhydramine, Pepcid, } \\
\text { hydrocortisione. } \\
\text { Pharmacy compounding PO analgesics: } \\
\text { codeine. Acetaminophen IV. } \\
\text { Fentanyl IV. }\end{array}$ & $\begin{array}{l}\text { Antibiotic: cefazolin IV. Instrumentation } \\
\text { included cages and allograft: cage filled } \\
\text { with local bone graft from osteophyte } \\
\text { shavings and demineralized bone } \\
\text { matrix. Hemostasis: Tisseel and Arista } \\
\text { (specific surgical powder); Tranexamic } \\
\text { acid IV. Suture: vicryl, steri-strips. }\end{array}$ \\
\hline 2 & $\begin{array}{l}\text { Laryngeal Mask, sevoflurane, and IV } \\
\text { agents: midazolam, fentanyl, lidocaine, } \\
\text { propofol. } \\
\text { Paracervical block with lidocaine } 1 \% \text { with } \\
\text { epinephrine. }\end{array}$ & Tora & n IV. \\
\hline 3 & $\begin{array}{l}\text { Isobaric spinal bupivicane after local } \\
\text { anesthetic skin wheal with lidocaine } 1 \% . \\
\text { IV agents: midazolam, fentanyl, propofol } \\
\text { infusion for sedation. }\end{array}$ & $\begin{array}{l}\text { ERAS PO agents: Celecocib, pregabalin, } \\
\text { and acetaminophen. } \\
\text { Intraoperative local Exparel injected } \\
\text { into knee compartments (by surgeon } \\
\text { protocol). } \\
\text { Oxycodone PO. } \\
\text { Hydromorphone IV. }\end{array}$ & $\begin{array}{l}\text { Antibiotic: cefazolin IV. Cement: Methyl } \\
\text { methacrylate. } \\
\text { Tranexamic acid IV. } \\
\text { Odansetron IV. } \\
\text { Suture: monocryl, vicryl, nylon. }\end{array}$ \\
\hline 4 & $\begin{array}{l}\text { Endotracheal tube, sevoflurane, and IV } \\
\text { agents: midazolam, fentanyl; lidocaine } \\
\text { and propofol boluses with infusions; } \\
\text { rocuronium, sugammadex. } \\
\text { Local anesthetic infiltration of marcaine. } \\
25 \% \text { with epinephrine at portal sites by } \\
\text { surgeon. }\end{array}$ & $\begin{array}{l}\text { ERAS PO agents: celecocib, gabapentin. } \\
\text { Preemptive IV agents: hydrocortisone, } \\
\text { diphenhydramine. } \\
\text { Desmopressin PO. } \\
\text { Acetaminophen IV. } \\
\text { Toradol IV. } \\
\text { Fentanyl IV. } \\
\text { Hydromorphone PO. } \\
\text { Hydromorphone IV. }\end{array}$ & $\begin{array}{l}\text { Antibotics: clindamycin, gentamicin. } \\
\text { Indigo carmine IV. } \\
\text { Odansetron IV. } \\
\text { Suture: vicryl. } \\
\text { Repeat desmopressin PO. } \\
\text { Repeat hydrocortisone IV. } \\
\text { Hydroxyzine PO. } \\
\text { Cetirizine PO. } \\
\text { Amitriptyline. } \\
\text { Buspirone. }\end{array}$ \\
\hline 5 & $\begin{array}{l}\text { Endotracheal tube, sevoflurane and IV } \\
\text { agents: Midazolam, fentanyl, lidocaine, } \\
\text { propofol, succinylcholine, } \\
\text { rocuronium, sugammadex. } \\
\text { Local anesthetic infiltration of marcaine. } \\
25 \% \text { with epinephrine at incision or portal } \\
\text { sites by surgeon. }\end{array}$ & $\begin{array}{l}\text { Acetaminophen IV. } \\
\text { Hydromorphone IV. }\end{array}$ & $\begin{array}{l}\text { Antibiotics: piperacillin/tazobactam } \\
\text { Suture: vicryl, monocryl, exofin. } \\
\text { Enoxaparin, subcutaneous prior } \\
\text { to surgery; repeated throughout } \\
\text { hospitalization. }\end{array}$ \\
\hline
\end{tabular}

ERAS: Enhanced Recovery After Surgery; IV: Intravenous; TIVA: Total Intravenous Anesthesia; PO: Oral. 
cist to compound his oral (PO) medications. Total intravenous anesthesia (TIVA) was required for intraoperative neurophysiology monitoring during major spine surgery. After judicious preoperative planning the patient received no triggering medications or surgical products. The patient tolerated the anesthesia and surgery well and his postoperative course was smooth using his personal compounded medication regime, including food preparation by the patient's spouse during his hospitalization. Pertinent social history in this case included he is a retired professional healthcare worker, as well as, volunteer worker on the Appalachian trail.

\section{Case 2}

Describes a patient identified by an immediate severe anaphylactic reaction to the cancer drug intravenous cetuximab. This patient only tolerated one generic brand of ibuprofen which she described as the Walmart brand. The patient did not receive routine enhanced recovery after surgery (ERAS) parenteral medications and her perioperative course was unremarkable. The carbohydrate containing drug cetuximab administered intravenously to patients with alpha-gal allergy can trigger immediate life-threatening anaphylaxis much different compared to the delayed onset of symptoms for those with MMA.

\section{Case 3}

Describes a patient with a history of delayed pruritis and urticaria following ingestion of beef containing meals on multiple occasions and confirmed alpha-gal allergy with formal allergy testing. She presented for total knee arthroplasty under neuraxial anesthesia and received the standard pre-emptive multimodal analgesic medications celecocib, pregabalin, and acetaminophen. No surgical hemostatic agents were required and the manufacturer of the methyl methacrylate bone cement confirmed its safety for use in this case. Her perioperative course was unremarkable and she was discharged to inpatient rehabilitation on postoperative day 2 , where she continued to recover without incident.

\section{Case 4}

Involves a patient who inadvertently received gabapentin as part of the ERAS protocol and before the alpha-gal allergy was determined and entered into the EHR. A swift internal query was underway to determine the manufacture of the pregabalin. However, the protective package contained only a lot number and no manufacturer's identification. Further inquiry through pharmacy led to a New Jersey website to locate the drug lot or medication distribution center. The patient demonstrated no signs or symptoms of allergic reaction within 6-12 hours after administration of the ERAS medication. The inquiry to locate the distribution center failed to provide a timely search to identify the actual manufacturing source.

\section{Case 5}

Describes a patient with confirmed alpha-gal allergy who underwent an emergent laparoscopy for gallstone ileus. She has regular allergist follow up and annual alpha-gal titers and
IgE levels drawn. While her levels had been elevated since the initial diagnosis in 2015, both alpha-gal and IgE drawn in 2018 were half of the titers compared to 2017. Before surgery, she received low dose enoxaparin $40 \mathrm{mg}$ for DVT prophylaxis, continued once a day throughout her hospital admission. Her perioperative course was unremarkable and she was discharged home without any anaphylactic episode during her hospitalization.

\section{Case 6}

Presents as an unusual casein a patient identified with a history of food allergy to beef and pork but the medical record did not highlight or specifically identify allergy to medications. Preoperatively, the EHR did not trigger or specify alpha-gal or MMA warning labels. She was not a candidate for an ERAS protocol and received no known triggering agents. She underwent elective gynecologic surgery without intraoperative concerns or issues. However, during her PACU stay she was given intravenous albumin for colloidal management of postoperative hypotension and developed immediate anaphylaxis. During the management of her allergic reaction the patient had been identified as having MMA by her primary care physician but neglected to present this as an important issue during the time of her Preoperative Care clinic appointment. Since the MMA was not clarified preoperatively appropriate perioperative planning was not conducted in the usual fashion for those patients with alpha-gal allergy. The case underwent (internal) review and although the patient was not exposed to any identifiable common triggering medications or surgical products the observation that a pooled blood product (derivative) such as human albumin remains suspect as the potential trigger for anaphylaxis (and as related to MMA with the sensitization mechanism undefined) in this patient. Authors present this case as a perioperative reoccurring event at our institution.

\section{Challenges in Anesthesia}

\section{Patient awareness}

The first challenge represents a knowledge gap, which includes patient awareness. Patients may have no knowledge or recall of tick exposure. Delayed response after exposure or sensitization by other mechanisms still not understood increases risk. Next, patients may not be aware of the alpha-gal allergy or its implication regarding medications or exposure to surgical products containing mammalian byproducts. Patient recognition having simply a food allergy to beef or pork may be informally acknowledged during an interview on the day of surgery. The day of surgery may be inadequate time to prepare for the patient dependent on the surgical procedure, anesthetic requirements, and postoperative disposition. Awareness by our Preoperative Care Clinics (PCC), surgical colleagues, anesthesia care teams, perioperative nursing is imperative to assure patient safety throughout the perioperative course. As increased awareness becomes more the norm the challenge gaps will improve throughout the system.

\section{Healthcare provider awareness}


Citation: D'Ercole FJ, Dhandha VH, Levi ML, et al. (2019) Perioperative Challenges in Patients with Alpha-Gal Allergy. J Clin Anesth Pain Manag 3(1):70-78

Table 3: Inactive compounds containing animal derivative and alpha-gal carbohydrate.

\begin{tabular}{|c|c|}
\hline Inactive compounds & Common perioperative drugs and materials containing inactive compounds \\
\hline Gelatin* & Acetaminophen tablet, celecoxib tablet, pregabalin tablet, gabapentin tablet, lidocaine patch, surgifoam \\
\hline Glycerin & Acetaminophen with codeine elixir, acetaminophen liquid, ibuprofen suspension, morphine solution \\
\hline Lactic Acid & Hydromorphone injection by Hospira \\
\hline Magnesium Stearate ${ }^{\dagger}$ & Acetaminophen tablet, oxycontin tablet, diphenhydramine tablet, lisinopril tablet \\
\hline Steric Acid ${ }^{+}$ & Oxycodone tablet by Hospira, Mallinckrodt \\
\hline
\end{tabular}

*Gelatin contains bovine and/or porcine. There are few exceptions such as gelatin containing plant-based products; ${ }^{\dagger}$ Stearate or steric acid is derived from bovine or plant sources.

Providers with access to populating the patient's medical records may not recognize signs, symptoms or trends. There is a clear disadvantage when specific questions are not entertained, such as, "Have you suffered from a tick bite?" Perioperative screening is important to enable proper management throughout the process. The first communication may actually start in the surgery clinic (or the surgeon's office) initiating the primary purpose of a PCC appointment. This includes recognizing and asking questions early regarding food allergies or medication allergies known to trigger the alpha-gal response.

Patients with a strong history of alpha-gal allergy will likely have an allergic response not limited to medications and may include intraoperative products such as catgut suture, bio-mesh, cardiac valves, grafts, thrombin, other topical hemostatic agents $[23,24]$. Other agents cited in the 2016 commentary by Commins include porcine or bovine derived heart valves, [25] high-dose heparin used during cardiac bypass, as well as, influenza vaccines, and the zoster vaccine. Therefore, early communication to anesthesia providers and critical operating room personnel may require several days or weeks to address inventory and prepare for patients requiring major surgery.

\section{Data collection and limitations of EHR}

Accurate or up-to-date information used to populate electronic healthcare records of patients with alpha-gal allergy may be misrepresented and under reported for several reasons. Limitations to electronic healthcare records (EHR) include the inability of physicians, and perioperative nursing to enter updated patient allergy information. At our institution, although the correct biochemical nomenclature alpha-gal can be entered into the EHR this terminology is less utilized by those entering the data.

Data entry of the more commonly used clinical terms most likely trigger an invalid entry. Terms such as alpha-gal allergy, beef allergy, mammalian, and red meat allergy may constitute invalid entries. Therefore, many medical records have this allergy unclassified or not accurately documented. If this allergy is not appropriately documented on the patient's allergy list then physician orders will not have the pharmacy safety-stops. Why is this important? It is considered a system failure when patient allergy lists are not updated and intrinsic safety-stops now customary in modern EHR cannot function properly. Patients scheduled for elective surgery may be assigned into ERAS protocols including pre- emptive medication protocols. Physicians placing most ERAS medication orders have previewed the patient's medical record but the alpha gal allergy is not properly cited in the EHR. If the allergy information is not accurate or timely then many pre-emptive medications if administered place the patient at risk for exposure to triggering agents based on the manufacturer's formulation (Table 3).

\section{Pharmacy information updates}

Prescribing medications throughout the perioperative course is a distinct challenge in patients with alpha-gal allergy. While formulations of drugs may vary between manufacturers, many oral medications may contain gelatin, glycerin products, magnesium stearate or stearate derivatives $[17,26-$ 28].

Authors recommend caution especially for patients enrolled in ERAS protocols outlining pre-emptive medications. At our institution physician-Allergists have compiled a drug list requiring continues active monitoring for manufacture's updates. Some institutions rely on the hospital's pharmacy to assure medication drug lists are accurate as described by Dunkman, et al. [17] In fact authors of this manuscript concur with Dunkman, et al. the importance in understanding the drug lists will vary between (our) institutions because inactive compounds containing animal derivative differ between manufacturers, as well as, drug lots having mixed suppliers. Examples of inactive compounds (Table 3 ) which may trigger alpha-gal allergy with the understanding the active ingredient is not the offensive component. Case 1 describes the anaphylaxis severity in a patient requiring a local pharmacy to compound all of his personal (PO) medications. Currently, manufacturers are not required to report alpha-gal content or changes in inactive ingredients.

\section{Access to pharmaceutical or manufacturers speci- fications}

There is a national drugcode (NDC) assigned to every medication clearly labeled on the protective wrap (Figure 2). This NDC number is used to identify the proprietary name, non-proprietary name, dosage form, and specific company of every medication.

To perform an advance search, see (https://ndclist.com/ search). However, anesthesia care teams and pharmacists at our institution may not be able to effectively identify medication tablets by drug lots. Drug lots may have a mix 


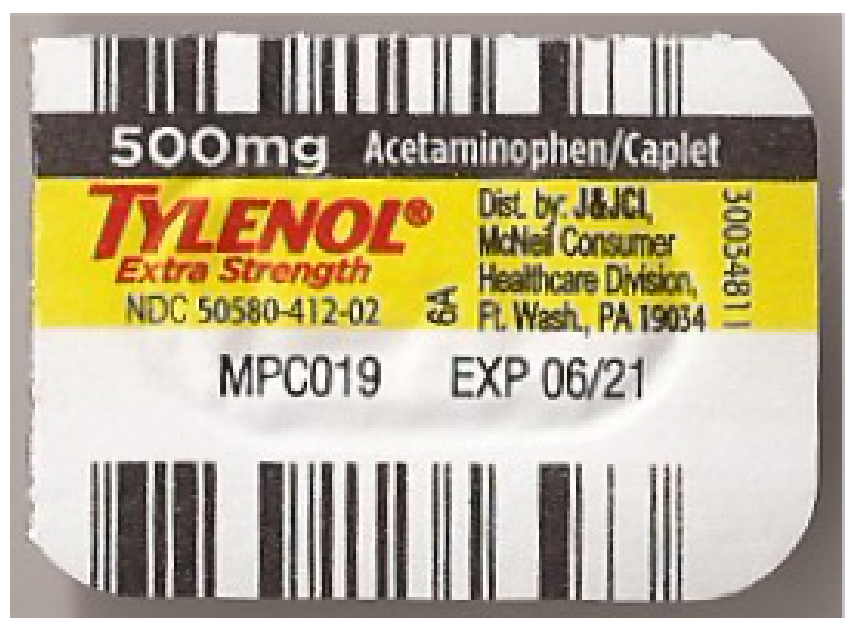

Figure 2: Drug manufacturer and the NDC number are clearly identified on the tablet.

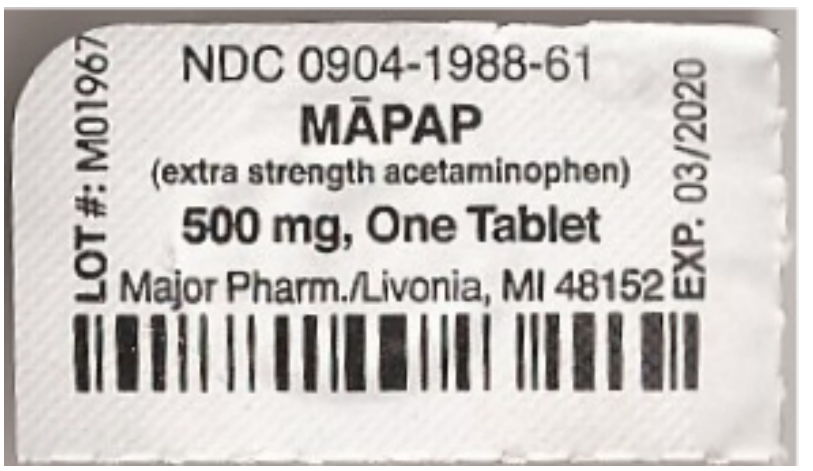

Figure 3: Generic tablet from unknown manufacturer displays both NDC and Lot number provided by supplier.

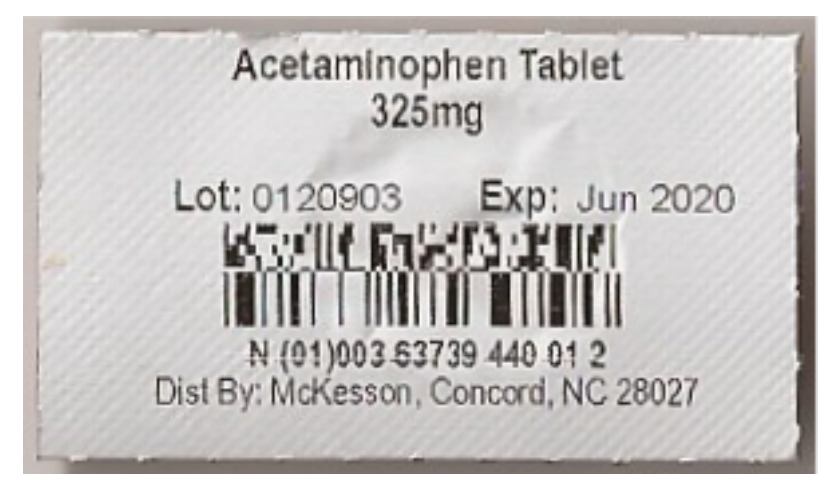

Figure 4: Generic tablet from unknown manufacturer displays the Lot number without a NDC number provided by the distributor.

of manufactures with a primary distribution center location in-state or out-of-state (Figure 3). There may be no direct access or communication to efficiently access drug lots, especially necessary on the day of surgery. Another example deviating from (NDC) norms includes a generic tablet from an unknown manufacturer displaying the Lot number without an NDC number provided by the distributor (Figure 4). These examples are problematic for hospitals and local pharmacies with limitations to updating drug lists for medications or compounds known to trigger the allergic response. Case 4 is an example of the limitations healthcare providers have to access important drug information.

\section{Demographics}

Currently the literature is weak describing alpha-gal allergy in the context of emerging tick-borne diseases. However, the incidence of many vector-borne infections has been on the rise and new infections have emerged. Changes in distribution and frequency of these infections may be among the first signs of the effect of global climate change on human health [29].

The eastern and southeastern region of the United States demonstrates the widest distribution of the vector Amblyomma americanum referred to as the Lone Star Tick [30].

\section{Blood supply}

To date, transfusion products in the perioperative period have not been identified as a method of sensitization for the alpha-gal allergy. However, updates by the CDC should be followed. Other considerations worthy of investigation (and remain suspect by our institution) include pooled products such as albumin or platelets. Although albumin is derived from human plasma it should be alpha gal safe, however, consideration should be given to the fact that there most likely exist other methods of sensitization as stated previously. Sell-Dottin, et al. have also suggested albumin not be given for resuscitation due to concern for alpha-gal reactivity during cardiac surgery [31].

The CDC has identified several tick-born illnesses such as Babesiosis and Lymes disease which have been associated with blood transfusion. Specific concern to these illnesses have been identified in the Northeast United States which have more likely than not contaminated blood supply in the US region with organisms causing Babesiosis and Lymes. Case 6 reminds us of the possibility of an indeterminate or unknown mechanism of sensitization for MMA (may exist).

\section{Prevention}

There is no cure for MMA. There is no vaccine. Prevention should be the priority particularly during the spring, summer, and early fall when ticks are most active [32]. The CDC outlines prevention methods including the avoidance of tick habitats. Attire should consist of light-colored clothing with long sleeves, long pants, and boots in tick-infested vegetation. The $\mathrm{CDC}$ recommends the use of insect repellant such as permethrin be applied to clothing [32]. There does exist a public health network, Tick NET. This network was created in 2007 to provide the collaboration between state health departments, academic centers, and the CDC to enhance surveillance and improve prevention of tick-borne diseases, including new tick-borne diseases [33].

\section{Conclusion}

Perioperative preparation for patients with known alpha-gal allergy requires necessary inquiry with pharmacists, 
drug manufacturers, and surgical product manufactures to confirm formularies, medication safety, and product specifications. The primary aim is avoiding or eliminating triggering agents. Common potential exposures of alpha-gal in the operating room include bio-prosthetic devices such as cardiac valves, surgical mesh products, hemostatic agents, and intravenous medications including but not limited to insulin and heparin. Heparin and heparin derivatives are likely to contain alpha-gal since they are porcine derived; however, there exists variability among different manufacture's drug lots. Triggering a reaction may be dependent on pharmaceutical processing or dose. Most of the reported heparin reactions have been associated with high-dose heparin commonly used during cardiac surgery [25]. Complex surgical procedures may require perioperative consultation well in advance to confirm safety specifications of the medications or products in question. Case 5 is an example of a patient managed with the heparin derivative - enoxaparin. Theoretically since enoxaparin is porcine derived the patient should be considered at increased risk for triggering a response, however, the drug dose was low and the patient's titers were low. Consideration should be given to the severity of the patient's symptoms or preoperative disposition. For example, Case 1 may not be a good candidate for a trial of low dose heparin or a heparin derivative based on his symptomatology. In retrospect, both Case 1 and Case 5 would have been good candidates for preoperative assay for alpha-gal titers and IgE levels.

Pre-anesthesia evaluation should include review of pre-existing known food and drug allergies, severity of symptoms after exposure, and historical effective treatment for prior symptoms. Previous records from allergy-immunology specialists including historical titers of alpha-gal and IgE are valuable sources to review prior to surgery as they often summarize past exposures and any progression of reactions. The intra-operative anesthetic plan may warrant general anesthesia with or without inhalational agents, neuraxial or regional anesthesia techniques to support effective postoperative pain management. Inhalational agents, intravenous induction agent propofol, local anesthetics, intravenous opioids have not been contraindicated in their present formulary. However, surgical products, which have been identified as triggers and are contraindicated include: Porcine-derived heart valves, xenografts, surgical powder, surgifoam powder, and thrombin. Therefore, it is imperative for the anesthesia care team, surgical team, and operating room nursing team to communicate concerns and inform all perioperative members prior to the elective scheduled case.

In addition to individual case analysis, group analysis was conducted for patients who did not manifest allergic reactions despite having an increase risk (Table 2b). was formulated listing anesthesia agents, analgesics, anesthesia and surgical modalities, surgical products, and miscellaneous therapeutic drugs during hospitalization. Authors hypothesize cases 1-5 who did not have a perioperative allergy-related complication may be due to hyper-vigilance by healthcare providers, including selectivity of medications and surgical products by anesthesia care teams and surgical teams. An example of hyper-vigilance may include the administration of preemptive diphenhydramine, dexamethasone or hydrocortisone cited in cases 1, 3, and 4. Group analysis identified cases 3-5 in patients who were at risk and either inadvertently or intentionally received triggering agents without complication. Cases 3 and 4 were included in ERAS protocols (including preemptive oral analgesics) governed by surgical teams having limitations to EHR information or pharmacy safety-stops.

One of the most common potential allergen exposures for patients presenting for surgery is the administration of oral medications. While formulations of drugs may vary between manufacturers, many oral medications may contain gelatin, glycerin products or stearate [26-28]. The possibility of allergen exposure must be considered carefully when implementing preemptive multimodal analgesic medications as part of ERAS pathways. These ERAS medication regimens may need to be reviewed for each individual drug and formulation to minimize the risk of potential exposure. Oral non-steroidal anti-inflammatory drug formularies in particular require rigorous review before subjecting patients to inadvertent (delayed) life-threatening exposure. Preemptive lists of perioperative medications and surgical products should include collaboration with pharmacy [17]. It is reasonable for patients and Preoperative Care Clinics to preemptively identify outpatient pharmacies offering compounding medications for postoperative requirements for those patients with alpha-gal allergy.

Finally, it is important clinicians grasp a better understand of MMA at the molecular level. Humans do not naturally produce the alpha-gal epitopes representing a molecular region on the surface of an antigen capable of eliciting an immune response. Instead humans produce Immunoglobulin G (IgG2) or anti-gal naturally protecting them from normal bacterial gut flora producing the alpha-gal epitope. Research suggests exposure to the alpha-gal epitope from a tick bite may cause normal anti-gal antibody formation to switch from IgG2 to IgE, causing the delayed anaphylaxis response to specific mammalian meat [34,35]. Patients with blood group $B$ or $A B$ have fewer anti-gal antibodies and therefore may be less likely to develop MMA or develop a milder reaction [35]. Present scientific literature suggests we are just scratching the surface of understanding MMA. Therefore, it is important to maintain high vigilance as clinical medicine and basic science researchers continue to find answers to fill present knowledge gaps.

\section{References}

1. Commins SP, Platts-Mills TA (2013) Delayed anaphylaxis to red meat in patients with IgE specific for galactose- $\alpha$-1,3-galactose (alpha gal). Curr Allergy Asthma Report 13: 72-77.

2. Commins SP, Platts-Mills TA (2009) Anaphylaxis syndromes related to a new mammalian cross-reactive carbohydrate determinant. J Allergy Clin Immunol 124: 652-657.

3. (2019) National Institute of Allergy and Infectious Diseases.

4. Oneil BH, Allen R, Spigel DR, et al. (2007) High incidence of cetuximab-related infusion reactions in Tennesse and North 
Carolina; association with atopic history. J Clin Oncol 25: 36443648.

5. Chung $\mathrm{CH}$, Mirakhur B, Chan E, et al. (2008) Cetuximab-induced anaphylaxis and IgE specific for galactose- $\alpha-1,3$-galactose. New Engl J Med 358: 1109-1117.

6. Commins SP, James HR, Kelly LA, et al. (2011) The relevance of tick bites to the production of IgE antibodies to the mammaliam oligosaccharide galactose- $\alpha$-1,3-galactose. J Allergy Clin Immunol 127: 1286-1293.

7. Centers for Disease Control and Prevention (2017) Tickborne Diseases of the United States: A Reference Manual for Health Care Providers, $\left(4^{\text {th }}\right.$ edn $)$.

8. (2019) Centers for Disease Control and Prevention. Geographic distribution of ticks that bite humans.

9. Hamsten C, Starkhammer M, Tran TA, et al. (2013) Identification of galactose- $\alpha$-1,3-galactose in the gastrointestinal tract of the tick Ixodes ricisnus: Possible relationship with red meat allergy. Allergy 68: 549-552.

10. Nuñez R, Carballada F, Gonzazlez-Quintel A, et al. (2011) Delayed mammalian meat-induced anaphylaxis due togalactose- $\alpha-1,3-$ galactose in 5 European patients. J Allergy Clin Immunol 128: 1122.e1-1124.e1.

11. Bircher AJ, Hofmeier HS, Link S, et al. (2017) Food allergy to the carbohydrate galactose-alpha-1,3-galactose (alpha-gal): Four case reports and a review. European Journal of Dermatology 27: 3-9.

12. Kennedy JL, Stallings AP, Platts-Mills TA, et al. (2013) Galactose$\alpha$-1,3-galactose and Delayed Anaphylaxis, Angioedema, Urticaria in Children. Pediatrics 131: 1545-1552.

13. Berg EA, Platts-Mills TA, Commins SP (2014) Drug allergens and food: The cetuximab and galactose- $\alpha$-1,3-galactose story. Ann Allergy Asthma Immunol 112: 97-101.

14. Stewart PH, McMiullan, KL, LeBlanc SB (2015) Delayed red meat allergy: Clinical ramifications of galactose- $\alpha$-1,3-galactose sensitization. Ann Allergy Asthma Immunol 115: 260-264.

15. Steinke JW, Platts-Mills TA, Comins SP (2015) The alpha-gal story: Lessons learned from connecting the dots. J Allergy Clin Immunol 135: 589-596.

16. Wilson JM, Schuyler AJ, Schroeder N, et al. (2017) Galactose$\alpha$-1,3-galactose: atypical food allergen or model IgE hypersensitivity? Curr Allergy Asthma Rep 17: 8.

17. Dunkman WJ, Rycek W, Manning MW (2018) What Does a Red Meat Allergy Have to Do With Anesthesia? Perioperative Management of Alpha-Gal Syndrome. Anesth Analg.

18. (2010) Viracor-IBT Laboratories Launches the First Assay to Identify a New Type of Delayed, IgE-based Allergic Reaction to Certain Meats. Viracor-IBT Laboratories.

19. Krishna Neal, Krishna S, Krishna R (2017) P112 Correlation between clinical findings and laboratory tests for alpha gal sensitivity. Ann of Allergy Asthma Immunol 119: S37.

20. Wolver Susan E, Sun Diane R, Commins Scott P, et al. (2013) A peculiar cause of anaphylaxis: No more steak? The journey to discovery of a newly recognized allergy to galactose-alpha-1,3galactose found in mammalian meat. Journal of General Internal Medicine 28: 322-325.

21. Jacquenet S, Moneret-Vautrin DA, Bihain BE (2009) Mammalian meat induced anaphylaxis: Clinical relevance of anti-galactosealpha-1,3-galactose IgE confirmed by means of skin tests to cetuximab. J Allergy Clin Immunol 124: 603-605.

22. Lee Jh, Kim JH, Kim TH, et al. (2013) Delayed mammalian meatinduced anaphylaxis confirmed by skin test to cetuximab. J Dermatology 40: 577-578.

23. Mozzicato SM, Tripathi A, Posthumus JB, et al. (2014) Porcine or bovine valve replacements in patients with IgE antibodies to the mammalian oligosaccharide galactose-alpha-1,3-galactose. J Allergy Clin Immunol Pract 2: 637-638.

24. Hawkins RB, Frischtak HL, Kron IL, et al. (2016) Premature bioprosthetic aortic valve degeneration associated with allergy to galactose-alpha-1,3-galactose. J Card Surg 31: 446-448.

25. Commins SP (2016) Invited Commentary: Alpha-gal Allergy: Tip of the Iceberg to a Pivotal Immune Response. Curr Allergy Asthma Rep 16: 61.

26. Mullins RJ, James H, Platts-Mills TA, et al. (2012) Relationship between red meat allergy and sensitization to gelatin and galactose- $\alpha-1,3-$ galactose. J Allergy Clin Immunol 129: 1334.e11342.e1.

27. Caponetto P, Fischer J, Biedermann T (2013) Gelatin-containing sweets can elicit anaphylaxis in a patient with sensitization to galactose- $\alpha$-1,3-galactose. J Allergy Clin Immunol Pract 1: 302-303.

28. Muglia C, Kar I, Gong M, et al. (2015) Anaphylaxis to medications containing meat by-products in an alpha-gal sensitized individual. J Allergy Clin Immunol Pract 3: 796-797.

29. Kovats RS, Haines A, Stanwell-Smith R, et al. (1999) Climate change and human health in Europe. BMJ 318: 1682-1685.

30. (2019) Centers for Disease Control and Prevention.

31. Sell-Dottin KA, Sola M, Caranaosos TG (2017) Impact of Newly Emerging Alpha-Gal Allergies on Cardiac Surgery: A Case Series. Clin Surg 2: 1477.

32. (2019) Centers for Disease Control and Prevention. Lyme and other tick borne diseases.

33. (2019) Centers for Disease Control and Prevention. TickNET-A Collaborative Public Health Approach to Tickborne Disease Surveillance and Research.

34. Galili U (2013) Anti-gal: An abundant human natural antibody of multiple pathogeneses and clinical benefits.Immunology 140 .

35. Rispens T, Derksen NI, Commins SP, et al. (2013) IgE production to $\alpha$-gal is accompanied by elevated levels of specific IgG1 antibodies and low amounts of IgE to blood group B. PLos One 8: e55566.

DOI: $10.36959 / 377 / 330$

Copyright: (c) 2019 D'Ercole FJ, et al. This is an open-access article distributed under the terms of the Creative Commons Attribution License, which permits unrestricted use, distribution, and reproduction in any medium, provided the original author and source are credited. 